\title{
Numerical Solution of Second-Orders Fuzzy Linear Differential Equation
}

\author{
Junyang An, Xiaobin Guo \\ College of Mathematics and Statistics, Northwest Normal University, Lanzhou, China \\ Email: guoxb@nwnu.edu.cn
}

How to cite this paper: An, J.Y. and Guo, X.B. (2021) Numerical Solution of SecondOrders Fuzzy Linear Differential Equation. Applied Mathematics, 12, 1118-1125. https://doi.org/10.4236/am.2021.1211071

Received: July 23, 2021

Accepted: November 27, 2021

Published: November 30, 2021

Copyright $\odot 2021$ by author(s) and Scientific Research Publishing Inc. This work is licensed under the Creative Commons Attribution International License (CC BY 4.0).

http://creativecommons.org/licenses/by/4.0/

\begin{abstract}
In this paper, the numerical solution of the boundary value problem that is two-order fuzzy linear differential equations is discussed. Based on the generalized Hukuhara difference, the fuzzy differential equation is converted into a fuzzy difference equation by means of decentralization. The numerical solution of the boundary value problem is obtained by calculating the fuzzy differential equation. Finally, an example is given to verify the effectiveness of the proposed method.
\end{abstract}

\section{Keywords}

Fuzzy Numbers, Fuzzy Differential Equations, Fuzzy Difference Equation, Fuzzy Approximate Solution

\section{Introduction}

Many engineering system problems are too complex to be directly converted into system equations to solve, and they often involve parameter uncertainties, which often appear as the fuzzy numbers. Therefore, when solving such problems, it is often necessary to model it to a fuzzy differential equation to consider, which always makes the solution of fuzzy system equations very important.

Prior to discussing fuzzy differential equations and their associated numerical algorithms, it is necessary to present an appropriate and brief introduction to the derivative of the fuzzy-valued function. The concept of a fuzzy derivative was first introduced by Zadeh [1], followed up by Dubois and Prade [2] who used the extension principle in their methods. Other fuzzy derivative concepts have been proposed by Puri and Ralescu [3] as an extension of the Hukuhara derivative of multivalued functions.

In recent years, many scholars have made profound research on two-order 
differential equations given the fuzzy boundary conditions. $\mathrm{Wu}, \mathrm{Q}$. [4] discussed the uncertainty of the two-point boundary value of the two-order differential equation. Using the fuzzy simulation principle and different methods, the numerical solution of the boundary value problem is obtained. Regan, O. et al. [5] proved a prior result on the solvability of fuzzy boundary value problems based on the generalized Schauder theorem. Wu, C.X. et al. [6] proved that the existence of analytical solutions of fuzzy boundary value problems completely depends on the definition, structure and properties of fuzzy numbers. Guo et al. [7] [8] studied the approximate solutions of two-order linear differential equations with several fuzzy boundary conditions. In this paper, we investigate the boundary value problem of two-order fuzzy linear differential equations.

\section{Preliminaries}

Definition 2.1 [1]: A fuzzy number is a fuzzy set like $u: R \rightarrow I=[0,1]$ which satisfies:

1) $u$ is upper semi-continuous;

2) $u(x)=0$ outside some interval $[c, d]$;

3) There are real number $a, b$ such that $c \leq a \leq b \leq d$ and a) $u(x)$ is monotonic increasing on $[c, a]$; b) $u(x)$ is monotonic decreasing on $[b, d]$; c) $u(x)=1, a \leq x \leq b$.

Definition 2.2 [2]: A fuzzy number $u$ in a parametric form is a pair $(\underline{u}, \bar{u})$ of functions $\underline{u}(r), \bar{u}(r), \quad 0 \leq r \leq 1$ which satisfies the following requirements: 1$)$ $\underline{u}(r)$ is a bounded monotonic increasing left continuous function, 2) $\bar{u}(r)$ is a bounded monotonic decreasing left continuous function, 3) $\underline{u}(r) \leq \bar{u}(r)$, $0 \leq r \leq 1$.

Definition 2.3 [1]: Let $x=(\underline{x}(r), \bar{x}(r)), y=(\underline{y}(r), \bar{y}(r)) \in E^{\prime}, 0 \leq r \leq 1$ and arbitrary $k \in R$, then:

1) $x=y$ i.f.f. $\underline{x}(r)=\underline{y}(r)$ and $\bar{x}(r)=\bar{x}(r)$;

2) $x+y=(\underline{x}(r)+\underline{y}(r), \bar{x}(r)+\bar{y}(r))$;

3) $x-y=(\underline{x}(r)-\bar{y}(r), \bar{x}(r)-\underline{y}(r))$;

4) $k x=\left\{\begin{array}{l}(k \underline{x}(r), k \bar{x}(r)), k \geq 0, \\ (k \bar{x}(r), k \underline{x}(r)), k<0 .\end{array}\right.$

Definition 2.4 [9]: Let $x, y \in E^{\prime}$. If there exists $z \in E^{\prime}$ such that $x=y+z$, then $z$ is called the Hukuhara difference of fuzzy numbers $x$ and $y$, and it is denoted by $z=x-y$.

Definition 2.5 [10]: Let $f:[a, b] \rightarrow E^{\prime}$ and $t_{0} \in[a, b]$. We say that $f$ is $\mathrm{Hu}-$ kuhara differential at $t_{0}$, if there exists an element $f^{\prime}\left(t_{0}\right) \in E^{\prime}$ such that for all $h>0$ sufficiently small, $\exists f\left(t_{0}+h\right)-f\left(t_{0}\right), f\left(t_{0}\right)-f\left(t_{0}-h\right)$ and the limits:

$$
\lim _{h \rightarrow 0} \frac{f\left(t_{0}+h\right)-f\left(t_{0}\right)}{h}=\lim _{h \rightarrow 0} \frac{f\left(t_{0}\right)-f\left(t_{0}-h\right)}{h}=f^{\prime}\left(t_{0}\right)
$$

Definition 2.6 [11]: The second-order fuzzy differential equation:

$$
\tilde{y}^{\prime \prime}=f\left(t, \tilde{y}, \tilde{y}^{\prime}\right), t \in[a, b]
$$


with the fuzzy boundary value conditions:

$$
\tilde{y}(a)=\tilde{\alpha}, \tilde{y}(b)=\tilde{\beta}, \tilde{\alpha}, \tilde{\beta} \in E^{\prime}
$$

is called the two-order fuzzy boundary value problems, where $\tilde{\alpha}=(\underline{\alpha}(r), \bar{\beta}(r))$.

In this paper, we mainly study the boundary value problem of two-order fuzzy linear differential equations, under the condition of parameter numbering.

$$
\left\{\begin{array}{l}
\tilde{y}^{\prime \prime}+p(t) \tilde{y}^{\prime}+q(t) \tilde{y}=g(t), t \in[a, b] \\
\tilde{y}(a)=\tilde{\alpha}, \tilde{y}(b)=\tilde{\beta},
\end{array}\right.
$$

where $\tilde{\alpha}, \tilde{\beta} \in E^{\prime}$ are fuzzy numbers, $p(t), q(t)$ are coefficient function. And parameter form is:

$$
\left\{\begin{array}{l}
\frac{y^{\prime \prime}(t, r)+p(t) y^{\prime}(t, r)+q(t) y(t, r)=g(t, r)}{\underline{y}(a, r)=\underline{\alpha}(r), \underline{y}(b, r)=\underline{\beta}(r)} \\
\frac{y^{\prime \prime}(t, r)+p(t) y^{\prime}(t, r)+q(t) y(t, r)=g(t, r)}{\bar{y}(a, r)=\bar{\alpha}(r), \bar{y}(b, r)=\bar{\beta}(r)}
\end{array}\right.
$$

where $t \in[a, b], 0 \leq r \leq 1$.

\section{The Establishment of the Difference Method}

For the boundary value problem of two-order fuzzy linear differential equations, we are going to discuss the establishment of the difference method and study its solvability in this section.

First, we use the following first-order difference quotient to approximate the first derivative $f^{\prime}(t)$, which is:

$$
\frac{y\left(t_{0}+h\right)-y\left(t_{0}\right)}{h} \approx y^{\prime}(t)
$$

or:

$$
\frac{y\left(t_{0}\right)-y\left(t_{0}-h\right)}{h} \approx y^{\prime}(t)
$$

or:

$$
\frac{y\left(t_{0}+h\right)-y\left(t_{0}-h\right)}{2 h} \approx y^{\prime}(t)
$$

Then the second derivative can be approximated by the first-order difference quotient of the first-order difference quotient, that is,

$$
y^{\prime \prime}(t) \approx \frac{y\left(t_{0}+h\right)-2 y\left(t_{0}\right)+y\left(t_{0}-h\right)}{h^{2}} .
$$

Let the integral interval $[a, b]$ be divided into $N$ equal parts, the step size is $h=\frac{b-a}{N}$, Its node is $N t_{n}=t_{0}+n h, n=0,1, \cdots, N$. Then use the difference quotient instead of the corresponding derivative, and the fuzzy differential boundary value problem (2.3) can be descreted into the following fuzzy difference problem. 


$$
\left\{\begin{array}{l}
\frac{\tilde{y}_{n+1}-2 \tilde{y}_{n}+\tilde{y}_{n-1}}{h^{2}}=\tilde{f}\left(t_{n}, y_{n}, \frac{y_{n+1}-y_{n-1}}{2 h}\right) \\
y_{0}=\tilde{\alpha}, y_{N}=\tilde{\beta}, \tilde{\alpha}, \tilde{\beta} \in E^{\prime}
\end{array}\right.
$$

According to the different $p(t)$ and $q(t)$ symbols, we discuss the solution of equation (3.1) from the following four case.

Case 1: $p(t)>0$ and $q(t)>0$, the difference form of the fuzzy boundary value problem (2.3) is:

$$
\left\{\begin{array}{l}
\underline{\underline{y_{n+1}}(r)-2 \overline{y_{n}}(r)+\underline{y_{n-1}}(r)}+p_{n} \frac{{\overline{y_{n+1}}}^{2}(r)-\overline{y_{n-1}}(r)}{2 h}+q_{n} \underline{y_{n}}(r)=\underline{g_{n}}(r) \\
\underline{y_{0}}(r)=\underline{\alpha}(r), \underline{y_{N}}(r)=\underline{\beta}(r) \\
\frac{\overline{y_{n+1}}(r)-2 \underline{y_{n}}(r)+\overline{y_{n-1}}(r)}{h^{2}}+p_{n} \frac{\overline{y_{n+1}}(r)-\underline{y_{n-1}}(r)}{2 h}+q_{n} \overline{y_{n}}(r)=\overline{g_{n}}(r) \\
\overline{y_{0}}(r)=\bar{\alpha}(r), \overline{y_{N}}(r)=\bar{\beta}(r)
\end{array}\right.
$$

Finishing the following the $2(N-1) \times 2(N-1)$ order matrix equations, in the form of:

$$
\left(\begin{array}{cc}
A_{1} & A_{2} \\
A_{2} & A_{1}
\end{array}\right)\left(\begin{array}{l}
\underline{Y}_{N-1}(r) \\
\bar{Y}_{N-1}(r)
\end{array}\right)=\left(\begin{array}{l}
\underline{B}_{N-1}(r) \\
\bar{B}_{N-1}(r)
\end{array}\right)
$$

where:

$$
\begin{aligned}
& A_{1}=\left(\begin{array}{ccccc}
q_{1} h^{2} & 1+\frac{h}{2} p_{1} & 0 & \ldots & 0 \\
1 & q_{2} h^{2} & 1+\frac{h}{2} p_{2} & \ldots & 0 \\
\vdots & \vdots & \ddots & \ddots & \vdots \\
0 & 0 & \ldots & q_{N-2} h^{2} & 1+\frac{h}{2} p_{N-2} \\
0 & 0 & \ldots & 1 & q_{N-1} h^{2}
\end{array}\right) \text {, } \\
& A_{2}=\left(\begin{array}{cccc}
-2 & 0 & \cdots & 0 \\
-\frac{h}{2} & -2 & \cdots & 0 \\
\vdots & \vdots & \ddots & 0 \\
0 & 0 & \cdots & -2
\end{array}\right) \\
& \underline{Y}_{N-1}(r)=\left(\underline{y}_{1}(r), \cdots, \underline{y}_{N-1}(r)\right)^{\mathrm{T}}, \bar{Y}_{N-1}(r)=\left(\bar{y}_{1}(r), \cdots, \bar{y}_{N-1}(r)\right)^{\mathrm{T}} \\
& \underline{B}_{N-1}(r)=\left(\underline{g}_{1}(r) h^{2}+\frac{h}{2} p_{1} \underline{\alpha}(r)-\bar{\alpha}(r), \underline{g}_{2}(r) h^{2}, \cdots, \underline{g}_{N-2}(r) h^{2},\right. \\
& \left.\underline{g}_{N-1}(r) h^{2}-\left(1+\frac{h}{2} p_{N-1}\right) \bar{\beta}(r)\right)^{\mathrm{T}} \\
& \bar{B}_{N-1}(r)=\left(\bar{g}_{1}(r) h^{2}+\frac{h}{2} p_{1} \bar{\alpha}(r)-\underline{\alpha}(r), \bar{g}_{2}(r) h^{2}, \cdots, \bar{g}_{N-2}(r) h^{2},\right. \\
& \left.\bar{g}_{N-1}(r) h^{2}-\left(1+\frac{h}{2} p_{N-1}\right) \underline{\beta}(r)\right)^{\mathrm{T}}
\end{aligned}
$$


Case 2: $p(t)<0$ and $q(t)<0$, the difference form of the fuzzy boundary value problem (2.3) is:

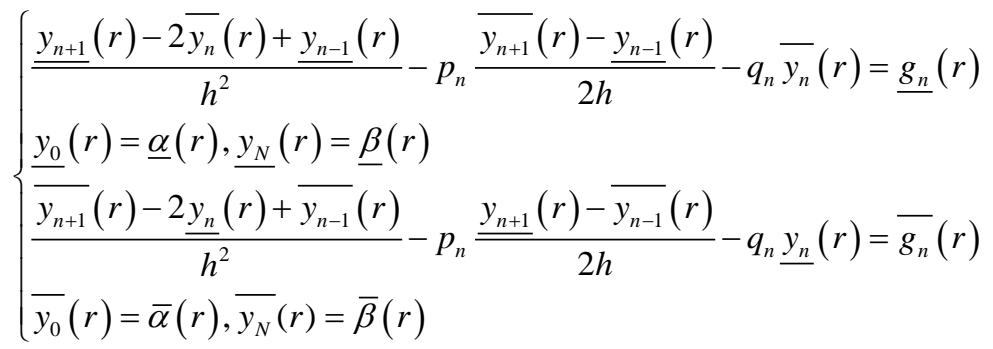

Finishing the following the $2(N-1) \times 2(N-1)$ order matrix equations, in the form of:

$$
\left(\begin{array}{ll}
C_{1} & C_{2} \\
C_{2} & C_{1}
\end{array}\right)\left(\begin{array}{l}
\underline{Y}_{N-1}(r) \\
\bar{Y}_{N-1}(r)
\end{array}\right)=\left(\begin{array}{l}
\underline{D}_{N-1}(r) \\
\bar{D}_{N-1}(r)
\end{array}\right)
$$

where:

$$
\begin{aligned}
& C_{1}=\left(\begin{array}{cccc}
0 & 1 & \cdots & 0 \\
1-\frac{h}{2} p_{1} & 0 & \cdots & 0 \\
\vdots & \vdots & \ddots & \vdots \\
0 & 0 & \cdots & 0
\end{array}\right), \\
& C_{2}=\left(\begin{array}{cccc}
-\left(2+q_{1} h^{2}\right) & -\frac{h}{2} p_{1} & \cdots & 0 \\
0 & -\left(2+q_{2} h^{2}\right) & \cdots & 0 \\
\vdots & \vdots & \ddots & \vdots \\
0 & 0 & \cdots & -\left(2+q_{N-1} h^{2}\right)
\end{array}\right) \text {, } \\
& \underline{D}_{N-1}(r)=\left(\underline{g}_{1}(r) h^{2}+\frac{h}{2} p_{1} \underline{\alpha}(r)-\bar{\alpha}(r), \underline{g}_{2}(r) h^{2}, \cdots, \underline{g}_{N-2}(r) h^{2}\right. \text {, } \\
& \left.\underline{g}_{N-1}(r) h^{2}-\left(1+\frac{h}{2} p_{N-1}\right) \bar{\beta}(r)\right)^{\mathrm{T}} \\
& \bar{D}_{N-1}(r)=\left(\bar{g}_{1}(r) h^{2}+\frac{h}{2} p_{1} \bar{\alpha}(r)-\underline{\alpha}(r), \bar{g}_{2}(r) h^{2}, \cdots, \bar{g}_{N-2}(r) h^{2},\right. \\
& \left.\bar{g}_{N-1}(r) h^{2}-\left(1+\frac{h}{2} p_{N-1}\right) \underline{\beta}(r)\right)^{\mathrm{T}}
\end{aligned}
$$

For Case 3: $p(t)<0$ and $q(t)>0$ and Case 4: $p(t)>0$ and $q(t)<0$, We can consider them by the same way.

Theorem 3.1 The solution of the fuzzy difference problem exists and is unique.

Proof First, we can eliminate the first-order difference in the fuzzy difference equation by appropriate transformation of the independent variables.

According to the results of the Negoita-Ralescu Characterization Theorem [12], 


$$
\left\{\begin{array}{l}
\underline{\underline{y_{n+1}}(r)-2 \overline{y_{n}}(r)+\underline{y_{n-1}}(r)}+q_{n} \underline{y_{n}}(r)=\underline{g_{n}}(r) \\
h^{2} \\
\underline{y_{0}}(r)=\underline{\alpha}(r), \underline{y_{N}}(r)=\underline{\beta}(r) \\
\overline{\frac{y_{n+1}}{1}(r)-2 \underline{y_{n}}(r)+\overline{y_{n-1}}(r)}+q_{n} \overline{y_{n}}(r)=\overline{g_{n}}(r) \\
\overline{h^{2}}(r)=\bar{\alpha}(r), \overline{y_{N}}(r)=\bar{\beta}(r)
\end{array}\right.
$$

Just prove that the corresponding homogeneous linear equations as:

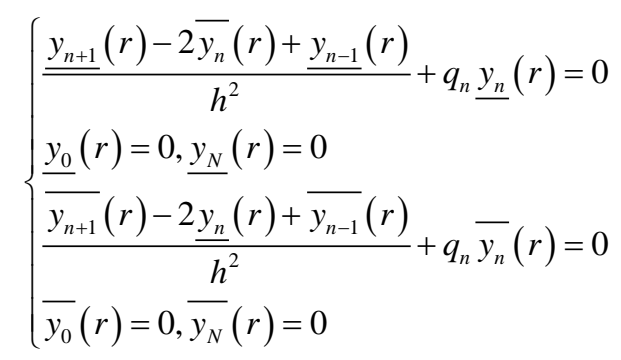

have only zero solutions. Obviously, the positive and negative minimum values of $\tilde{y}_{n}$ can only be $\tilde{y}_{0}$ or $\tilde{y}_{N}$ Also known by the boundary condition $\tilde{y}_{0}=\tilde{y}_{N}=[0,0]$, all $\tilde{y}_{n}=[0,0]$.

\section{Numerical Example}

Example 4.1 Consider the boundary value problem of fuzzy differential equations as follows:

$$
\left\{\begin{array}{l}
\tilde{y}^{\prime \prime}-\tilde{y}=t, t \in[0,1] \\
\tilde{y}(0)=(0.1-0.1 r,-0.1+0.1 r) \\
\tilde{y}(1)=(-0.1 r, 1+0.1 r)
\end{array}\right.
$$

The exact solution is follows:

$$
\left\{\begin{array}{l}
\underline{Y}(t, r)=(0.1-0.1 r) \cos (t)+(-0.1 r) \sin (t)-t \\
\bar{Y}(t, r)=(-0.1+0.1 r) \cos (t)+(1+0.1 r) \sin (t)-t
\end{array}\right.
$$

Converting the boundary value problem of fuzzy differential equations into boundary value problems of fuzzy difference equations, take the step size of 0.2 , the node $t_{n}=\frac{n}{5},(n=0,1,2,3,4,5)$, then its matrix form is:

$$
\left(\begin{array}{cccccccc}
0 & 1.1 & 0 & 0 & -1.96 & 0 & 0 & 0 \\
1 & 0 & 1.1 & 0 & 0 & -1.96 & 0 & 0 \\
0 & 1 & 0 & 1.1 & 0 & 0 & -1.96 & 0 \\
0 & 0 & 1 & 0 & 0 & 0 & 0 & -1.96 \\
-1.96 & 0 & 0 & 0 & 0 & 1.1 & 0 & 0 \\
0 & -1.96 & 0 & 0 & 1 & 0 & 1.1 & 0 \\
0 & 0 & -1.96 & 0 & 0 & 1 & 0 & 1.1 \\
0 & 0 & 0 & -1.96 & 0 & 0 & 1 & 0
\end{array}\right)\left(\begin{array}{l}
\underline{y}_{1} \\
\underline{y}_{2} \\
y_{3} \\
\underline{y}_{4} \\
\bar{y}_{1} \\
\bar{y}_{2} \\
\bar{y}_{3} \\
y_{4}
\end{array}\right)=\left(\begin{array}{c}
0.092-0.1 r \\
0.016 \\
0.024 \\
0.032 \\
0.092-0.1 r \\
0.016 \\
0.024 \\
0.032
\end{array}\right)
$$


Then, the solution of the boundary value problem of fuzzy differential equations is:

$$
\left\{\begin{array}{l}
\underline{Y}(t, r)=(0.045+0.100 r)+(-0.157+0.083 r) t+(-0.032+0.060 r) t^{2}+(-0.090+0.030 r) t^{3} \\
\bar{Y}(t, r)=(-0.135+0.100 r)+(-0.003+0.083 r) t+(-0.142+0.060 r) t^{2}+(-0.033+0.030 r) t^{3}
\end{array} .\right.
$$

\section{Conclusion}

In this paper, an approximate method based on a positive basis for the undetermined coefficients second-orders fuzzy linear boundary value problems was discussed. A class of boundary condition and the general case were considered. According to the sign of coefficient functions of the fuzzy linear differential equation, the corresponding function systems of linear equations were composed. And then, fuzzy approximate solutions were obtained by solving a crisp function extended system of linear equations. For next investigation, we can consider the other class of boundary conditions by the same method.

\section{Acknowledgements}

This work is supported by the National Natural Science Fund of China (No. 61967014, No. 11861059) and the Scientific Research Project of Gansu Province Colleges and Universities (No. 2019A-004).

\section{Conflicts of Interest}

The authors declare no conflicts of interest regarding the publication of this paper.

\section{References}

[1] Zadeh, L.A. (1965) Fuzzy Sets. Information and Control, 8, 338-353. https://doi.org/10.1016/S0019-9958(65)90241-X

[2] Dubois, D. and Prade, H. (1978) Operations on Fuzzy Numbers. International Journal of Systems Science, 9, 613-626. https://doi.org/10.1080/00207727808941724

[3] Puri, M.L. and Dan, A.R. (1983) Differentials of Fuzzy Functions. Journal of Mathematical Analysis and Applications, 91, 552-558. https://doi.org/10.1016/0022-247X(83)90169-5

[4] Wu, Q. (2001) The Fuzzy Boundary Value Problem for Differential Equation of Second Order Based on the Difference Method. Journal of National University of Defense Technology, 23, 40-43.

[5] O’Regan, D., Lakshmikantham, V. and NietoJ, J. (2003) Initial and Boundary Value Problems for Fuzzy Differential Equations. Nonlinear Analysis. Theory, Methods and Applications, 54, 405-415. https://doi.org/10.1016/S0362-546X(03)00097-X

[6] Chen, M., Wu, C., Xue, X., et al. (2008) On Fuzzy Boundary Value Problems. Information Sciences, 178, 1877-1892. https://doi.org/10.1016/j.ins.2007.11.017

[7] Guo, X., Shang, D. and Lu, X. (2013) Fuzzy Approximate Solutions of Second-Order Fuzzy Linear Boundary Value Problems. Boundary Value Problems, 2013, Article No. 1. https://doi.org/10.1186/1687-2770-2013-212

[8] Guo, X. and Gong, Z. (2013) Fuzzy Approximate Solution of Second Order Linear 
Differential Equations with Fuzzy Boundary Values. Fuzzy Systems and Mathematics, 27, 85-93.

[9] Bede, B. (2008) Note on "Numerical Solutions of Fuzzy Differential Equations by Predictor-Corrector Method”. Information Science, 178, 1917-1922. (In Chinese) https://doi.org/10.1016/j.ins.2007.11.016

[10] Wu, C.X. and Ma, M. (1991) Embedding Problem of Fuzzy Numbers Space: Part I. Fuzzy Sets and Systems, 44, 33-38. https://doi.org/10.1016/0165-0114(91)90030-T

[11] Bede, B. and Gal, S.G. (2005) Generalizations of the Differentiability of Fuzzy-NumberValued Functions with Applications to Fuzzy Differential Equations. Fuzzy Sets and Systems, 151, 581-599. https://doi.org/10.1016/j.fss.2004.08.001

[12] Negoita, C.V. and Ralescu, D.A. (1975) Application of Fuzzy Sets to System Analysis. Wiley, New York. https://doi.org/10.1007/978-3-0348-5921-9 